\title{
3 Research Square

\section{Tumor Abnormal Protein Detection is An Effective Screening Method for Solid Malignancies}

\section{Zhihui Zhang}

Second Affiliated Hospital of Soochow University

\section{Changjun Tian}

Second Affiliated Hospital of Soochow University

\section{Lin Zhang}

Second Affiliated Hospital of Soochow University

\section{Linfang Li}

Second Affiliated Hospital of Soochow University

\section{Mingxin Gao}

Second Affiliated Hospital of Soochow University

\section{Bai Li}

Second Affiliated Hospital of Soochow University

\section{Hui Fan}

Second Affiliated Hospital of Soochow University

\section{Yongsheng Zhang}

Second Affiliated Hospital of Soochow University

shuhua wu ( $\square$ wushuhuasz@163.com)

Second Affiliated Hospital of Soochow University https://orcid.org/0000-0002-5480-9126

\section{Research article}

Keywords: Tumor abnormal protein (TAP), Solid malignancies, Screening, Following-up.

Posted Date: May 4th, 2021

DOI: https://doi.org/10.21203/rs.3.rs-484071/v1

License: (c) (1) This work is licensed under a Creative Commons Attribution 4.0 International License. Read Full License 


\section{Abstract}

Background: Detection of tumor abnormal protein (TAP) is a recently developed but not widely used method for screening malignancies. This study is aimed to assess the ability of TAP level in distinguishing solid malignancies.

Methods: The TAP levels were compared between malignancies and benign disease patients, and the distinguish ability were evaluated by receiver operating characteristic (ROC) curve analysis. We further analyzed TAP levels in physical examination populations with different genders and age $\mathbb{Z}$ and compared clinical parameters in TAP positive and negative groups.

Results: We found that TAP levels were getting higher from benign to precancerous lesions and to malignancies, and TAP levels were decreased after tumor resection. ROC curve analysis showed that TAP levels might aid to distinguish between malignancies and benign diseases. In the physical examination populations, the positive ratio of TAP was $3.7 \%$ (4.6\% to female and $3.1 \%$ to male), and the TAP levels were higher in female. The TAP levels in populations above 60 year age were higher than those in less than 60 years, which increased with age. The hemoglobin levels were lower in TAP positive populations.

Conclusion: TAP was an effective biomarker of screening and following-up for malignancies. There may be some potential links between TAP and hemoglobin, which deserve further studies.

\section{Introduction}

Tumor abnormal protein (TAP) is a complex of glycoprotein and calcium-histone released by tumor cells during metabolism, and is considered as the biomarker among solid malignancies ${ }^{[1,2]}$. TAP is different from traditional tumor biomarkers which were only detected for specific tumor biomarkers in specific tumors. By detecting a variety of tumor-associated abnormal sugar chains in peripheral blood, more tumor signals can be aggregated at high concentrations ${ }^{[3]}$. Due to convenient detection, sensitivity and broad spectrum, TAP detection has been routinely performed to many hospitalized patients, as well as in physical examination population. At present, some reports ${ }^{[4,5,6]}$ demonstrated that TAP was closely related to a variety of tumors. $\mathrm{Ma}$, et $\mathrm{al}^{[4]}$ found that TAP combined with transvaginal ultrasound had a higher accuracy for the diagnosis of early endometrial cancer. Zhang et al ${ }^{[5]}$ found that TAP contributed to the initial diagnosis of bladder cancer. Li et al ${ }^{[6]}$ found the overall survival rates of patients with low expression of TAP in pancreatic, gallbladder, bile duct, and liver cancers were significantly elevated than patients with high expression of TAP. TAP is now being gradually applied for early detection of cancer. However, there are still some uncertainties for this diagnostic method. Therefore, this study will evaluate serum TAP detection in diagnosis of solid malignancies.

In clinic, the diagnosis, treatment and prognosis assessment of malignancies are increasingly personalized ${ }^{[7,8]}$. Therefore, searching differences in individuals from populations was an important direction for development of medical therapy. The development of precision medicine is facilitated by the 
development of diagnostic criteria according to the characteristics of different populations. In clinical practice, there are many laboratory parameters that select different reference ranges to diagnose different populations, such as the level of hemoglobin among populations with different genders ${ }^{[9,10]}$. This study compared the TAP levels among different populations to determine the population heterogeneity, and to provide the new bases for accurate diagnosis.

\section{Materials And Methods}

\section{Research population}

The populations enrolled in this study were the inpatients of the General Medical Department and the physical examination subjects of Physical Examination Center in the Second Affiliated Hospital of Soochow University from October 2017 to June 2019. All the subjects have completed the detection of TAP, and these with unclear diagnosis and incomplete data were not enrolled in the study. There were 638 cases of inpatients, including 438 cases of benign diseases, 24 cases of precancerous lesions, and 176 cases of malignancies. A total of 630 physical examination subjects were enrolled, including 392 males and 238 females. 89 subjects were older than or equal to 60 years old, and 541 cases were younger than 60 years old(Table 1). In this study, inpatients were selected to study the distinguishing ability of TAP, and physical examination subjects were selected to discover the heterogeneity of TAP.

Table 1

Number of inpatients and physical examination people

\begin{tabular}{|llllll|}
\hline & Gender & & Age & & Total \\
\hline $\begin{array}{l}\text { Physical examination } \\
\text { subjects }\end{array}$ & Female & Male & $\geq 60$ years & $\begin{array}{l}<60 \\
\text { years }\end{array}$ \\
\cline { 2 - 5 } & 238 & 392 & 89 & 541 & 630 \\
\hline Inpatients & Diseases & & & Total \\
& $\begin{array}{l}\text { Benign } \\
\text { diseases }\end{array}$ & Precancerous lesions & malignancies & \\
\hline 438 & 24 & 176 & 638 \\
\hline
\end{tabular}

\section{Tap Detection}

TAP detection is a clinical standard test in the Second Affiliated Hospital of Soochow University hospital, and the price is about US\$75. Acquisition $2 \mathrm{ml}$ peripheral blood from fingertips, then made two pieces of blood smear, dry for 10min, 3 drops of TAP reagent (Zhejiang Ruisheng Medical Technology Co., Ltd.) about 150ul were dripped on the blood smear. A coagulation staining reaction was performed and produced aggregated particles. The TAP aggregated particles were measured with a $40 x$ objective lens by two pathologists. 


\section{Inpatients}

According to the diagnostic criteria of World Health Organization (WHO) ${ }^{[11-15]}$, inpatients who had received TAP test were divided into benign disease group, precancerous lesion group and malignant tumor group. The diagnostic criteria for precancerous lesions were based on pathological detection with standard procedure, including atypical hyperplasia of esophageal squamous cells and Barrett's esophagus, gastrointestinal metaplasia, intraepithelial neoplasia and heterosexual hyperplasia, atypical hyperplasia of mammary duct and lobular epithelium, and intraepithelial neoplasia, tubular adenoma, heterosexual hyperplasia in colorectum, atypical hyperplastic nodules in liver, cervical intraepithelial neoplasia, and so on; and the diagnose of malignancies were based on the pathological detection with malignant cells and/or imaging evidence. All benign disease patients were excluded from malignancies by high-resolution computerized tomography (CT) or magnetic resonance imaging (MRI). There were no special requirements for the disease state and treatment stage of all patients in this study. The postoperative follow-up patients were completely remission according imaging diagnosis, and no special requirement for postoperative adjuvant treatment.

\section{Physical Examination Subjects}

In order to avoid the influence of subjective factors, we adopted the double-blind method when analyzing the heterogeneity of TAP levels in physical examination subjects. Among all 630 physical examination subjects, only 23 had positive TAP. 24 subjects were selected from remaining 607 people as TAP-negative group using randomly stratified sampling. The following parameters of physical examination subjects were included: age, gender, results of chest CT, tumor biomarkers (alpha-fetoprotein (AFP), carcinoembryonic antigen (CEA), ferritin, saccharide antigen CA125, saccharide antigen CA153, saccharide antigen CA199, saccharide antigen CA242, total prostate specific antigen (t-PSA), free prostate specific antigen (f-PSA), cytokeratin 19 fragment (CYFRA211), neuron specific enolase (NES), precursor of gastrin releasing peptide (ProGRP), human chorionic gonadotropin (HCG), human growth hormone $(\mathrm{HGH})$ ) and laboratory parameters (hemoglobin, white blood cell and platelet). We further analyzed differences of pulmonary nodule, tumor biomarkers and laboratory parameters between the two groups.

\section{Statistical Methods}

The SPSS 20.0 software package (SPSS, USA) was utilized for the statistical analysis. Mann-Whitney's U test were performed for continuous variables, and categorical variables were analyzed using Pearson chisquare analysis or Fisher's exact test. The receiver operating characteristic (ROC) curve and the area under the ROC curve (AUC) were used to test the ability of TAP levels in distinguishing malignancies and benign diseases patients. Spearman correlation test was performed for correlation analysis of 
quantitative data. For all statistics, a two-tailed $P$ value less than 0.05 was determined as statistically significant.

\section{Results}

\section{The levels of TAP detection in inpatients}

The levels of TAP in patients with malignancies [median 187 (range 80-402)] were significantly higher than those in patients with benign disease [median 101 (range 41-305), $P<0.001$, Fig. 1]. In precancerous lesions group, the TAP levels [median 161.5 (range 69-183)] were significantly higher than those of benign disease group, and lower than those of malignancies group $(P=0.017, P<0.001$, respectively, Fig. 1).

\section{Distinguish between malignancies and benign diseases patients with TAP levels}

ROC curve analysis showed that TAP levels had significant differences between malignancies and benign diseases (AUC $=0.922,95 \%$ confidence interval $(C I)=0.898-0.947, P<0.001$, Fig. 2 ). When the cut-off value of TAP level was 124.50, the Yoden index (sensitivity + specificity -1) was the largest and the maximum in terms of discrimination ability. Clinically, the TAP level was defined as $121 \mu \mathrm{m}^{2}$ to serve as the diagnosis standard from tumors, which was very close to our statistical results. Whereas clinical diagnostic criteria were derived from studies with larger sample sizes, this study still using $121 \mu \mathrm{m}^{2}$ to divide the population into TAP-positive and TAP-negative. The value of TAP levels more than $121 \mu \mathrm{m}^{2}$ were defined as TAP-positive, and in contrast 0-121.00 $\mu^{2}$ defined as TAP-negative.

\section{Monitoring Of Tap Level In Follow-up Malignancies Patients}

We had randomly followed up 10 newly diagnosed patients, who were candidates for surgical procedures, and found that TAP level was significantly declined from the initial diagnosis to post-surgical operation. $(P<0.001$, Fig. 3).

\section{Tap Positive Ratio In Physical Examination Subjects}

There were 23 of the 630 physical examination subjects whose TAP levels were greater than $121 \mu \mathrm{m}^{2}$, and the positive ratio was $3.7 \%$. The physical examination subjects were divided into male and female groups. In the male group, there was a positive ratio of $3.1 \%$, including 12 cases in all 392 male physical examination subjects; and in the female groups, there was a positive ratio of $4.6 \%$, including 11 of 238 female physical examination subjects. In contrast to male physical examination populations, TAP levels (female median 94 (range 59-148) vs. male median 87.00 (range 57-144), $P=0.001$, Fig. 4) in female were significantly higher. As for age factor, the TAP levels in subjects of $>60$ year old were higher than 
those in less than 60 years (median 87.00 (range 57-148), $P<0.001$, Fig. 4). Further analysis revealed that TAP levels increased with age in the physical examination subjects (Fig. 4).

\section{Comparison of pulmonary nodules in TAP positive and negative subjects in physical examination}

Routine physical examination items, usually including chest XCT scan, were performed in the Second Affiliated Hospital of Soochow University. This study showed that the positive ratio of pulmonary nodules was $94 \%(17 / 18,6$ patients without data of chest CT) in the TAP-positive group, and $67 \%(16 / 24)$ in the TAP-negative group,which declared the increasing trend in the TAP positive group $(P=0.055)$. The number of pulmonary nodules was 2.5 (range 1-7) in TAP-positive people, and was 2 (range 1-6) in TAPnegative people. The media size of largest pulmonary nodules was 7 (range 5-11) mm in TAP-positive people and was $6 \mathrm{~mm}$ (range 5-10) in TAP-negative patients. However, there was no statistically significant difference between the two groups in terms of the number or size of pulmonary nodules $(P=$ 0.201 and $P=0.615)$.

\section{Correlation Between Tap And Tumor Biomarkers}

From 23 TAP-positive and 24 TAP-negative subjects, tumor biomarkers were collected, and then analysis were performed. There was no statistical difference between TAP levels and tumor biomarkers, such as CA199 ( $\mathrm{R}=-0.063, P=0.673)$, NES $(\mathrm{R}=0.027, P=0.856)$, CEA $(\mathrm{R}=0.057, P=0.706), \mathrm{CA} 242(\mathrm{R}=0.103, P=$ 0.611). Fer ferritin ( $\mathrm{R}=-0.020, P=0.897)$, HCG $(\mathrm{R}=0.188, P=0.414)$, AFP $(\mathrm{R}=0.028, P=0.851)$, f-PSA $(\mathrm{R}=$ $0.210, P=0.302)$, t-PSA $(\mathrm{R}=-0.067, P=0.704), \mathrm{CA} 125(\mathrm{R}=0.218, P=0.146), \mathrm{HGH}(\mathrm{R}=0.311, P=0.170)$, CA153 $(\mathrm{R}=0.058, P=0.748)$, ProGRP $(\mathrm{R}=-0.162, P=0.440)$ and CYFRA211 $(\mathrm{R}=0.044, P=0.835)$.

\section{Comparison of laboratory parameters between TAP positive and negative groups}

Several laboratory parameters were tested and compared between the TAP positive to TAP negative groups. The hemoglobin levels [141 (range 98-175)] in TAP positive group were significantly lower than those in TAP negative group [155 (range 127-174)], and the difference between them was statistically significant $(P=0.040$, Table 2). There was no significant difference in white blood cell levels, platelet levels, and tumor marker levels between the two groups $(P>0.05$, Table 2$)$. 
Table 2

Comparison of laboratory data for TAP positive and negative patients

\begin{tabular}{|llll|}
\hline Parameters & TAP $(+)(\mathrm{N}=23)$ & TAP(-)(N=24) & P value \\
\hline WBC $\left(\times 10^{9} / \mathrm{L}\right)$ & $6(3.9-8.4)$ & $5.7(4-8)$ & 0.837 \\
\hline HB $(\mathrm{g} / \mathrm{L})^{\square}$ & $141(98-175)$ & $155(127-174)$ & 0.040 \\
\hline PLT $\left(\times 10^{9} / \mathrm{L}\right)$ & $232(148-365)$ & $231(135-274)$ & 0.716 \\
\hline AFP $(\mathrm{ng} / \mathrm{ml})$ & $0.93(0.24-6.29)$ & $0.4(0.24-4.55)$ & 0.756 \\
\hline CEA $(\mathrm{ng} / \mathrm{ml})$ & $1.29(0.23-5.30)$ & $1.32(0.39-4.12)$ & 0.992 \\
\hline CA125 $(\mathrm{U} / \mathrm{ml})$ & $6.34(1.69-12.65)$ & $7.225(2.30-11)$ & 0.125 \\
\hline CA153 $(\mathrm{U} / \mathrm{ml})$ & $5.98(0.53-19.74)$ & $5.6(3.19-20.06)$ & 0.899 \\
\hline CA199 $(\mathrm{U} / \mathrm{ml})$ & $7.05(0.6-44.77)$ & $6.645(2-19.9)$ & 0.748 \\
\hline CA242 $(\mathrm{U} / \mathrm{ml})$ & $2.1(1-3.63)$ & $2.11(1.46-2.67)$ & 0.394 \\
\hline NES $(\mathrm{ng} / \mathrm{ml})$ & $9.22(1.43-26.14)$ & $9.27(1-15.39)$ & 0.896 \\
\hline Fer-ferritin $(\mathrm{ng} / \mathrm{ml})$ & $140.9(7-396.1)$ & $82(13.5-362.1)$ & 0.955 \\
\hline HCG $(\mathrm{ng} / \mathrm{ml})$ & $0.19(0.02-0.34)$ & $0.085(0.01-0.63)$ & 0.257 \\
\hline HGH $(\mathrm{ng} / \mathrm{ml})$ & $0.11(0.01-3.11)$ & $0.045(0.01-0.25)$ & 0.319 \\
\hline t-PSA $(\mathrm{ng} / \mathrm{ml})$ & $0.37(0.04-4.29)$ & $0.16(0.04-1.40)$ & 0.709 \\
\hline f-PSA $(\mathrm{ng} / \mathrm{ml})$ & $0.07(0.02-1.07)$ & $0.085(0.02-0.45)$ & 0.533 \\
\hline ProGRP $(\mathrm{ng} / \mathrm{ml})$ & $30.675(21.3-55.94)$ & $37.12(17.8-47.5)$ & 0.295 \\
\hline CYFRA211 $(\mathrm{ng} / \mathrm{ml})$ & $2.34(0.98-12.77)$ & $2.27(1.61-3.58)$ & 0.810 \\
\hline The difference was statistically significant $(P<0.05)$ & \\
\hline
\end{tabular}

\section{Discussion}

For malignancies, early detection, early diagnosis and early treatment are important means of prevention and treatment. Physical examination and effective screening methods have been proven to be able to effectively reduce the social burden of malignancies ${ }^{[16,17]}$. From 1990 to 2015 , the overall cancer mortality rate fell $25 \%$ in the United States, which can be attributed to the introduction of high-quality cancer screening and the emergence of new screening methods ${ }^{[18]}$. TAP, with the classification standard of $121 \mu \mathrm{m}^{2}$, has been used as detection index of malignancies in clinic. This study evaluated the diagnostic ability of TAP level, analyzed the heterogeneity among different populations, and further 
investigated its relevance with clinical parameters for exploring more mechanisms. The results will be discussed in detail, respectively.

The accurate standard for malignancies screening is pathological examination, but it has limitation and is not suitable for most people. Therefore, indirect screening is still the most used diagnostic method in clinic ${ }^{[19]}$. This study showed our TAP level results were statistically consistent with the clinical standards, preliminary indicating that the method was reliable. We found the levels of TAP in malignancies patients were higher than those in precancerous lesion patients, and so on in precancerous lesion and benign patients. As the host cells became cancerous, TAP levels gradually increased, which suggested that TAP level could not only indicate the occurrence of malignancies, but also predict the development of malignancies. Further research showed that TAP level could be used as indicators to separate malignancies from benign diseases. Currently, our hospital recommended the cut off value to be $121 \mu \mathrm{m}^{2}$ as a parameter to distinguish TAP positive and TAP negative. In this study, the ROC curve analysis showed that the cut-off value could be 124.5, which has the highest sensitivity and specificity. The difference may be due to the different states of tumor patients, the limited sample size, and the population heterogeneity. Following up newly diagnosed patients, we found that the levels of TAP were decreased significantly after surgery of removing the tumors in these patients. This further indicated the TAP index had exceptional sensitivity to monitor malignancies.

Indirect malignancies screenings always accompanied with impaired screening effectiveness due to a decline in the performance characteristics including false positives and false negatives ${ }^{[20]}$. In this study, we analyzed population heterogeneity in the physical examination subjects. Our result showed that the positive ratio of TAP was 3.7\%, including 3.1\% male and $4.6 \%$ female. According to the American Cancer Society's 2019 report ${ }^{[21]}$ on the incidence of malignancies from 1975 to 2015 , the total incidence of malignancies in the United States in 2015 was about 425/100,000, including about 460/100,000 men and about 410/100,000 women, which was obviously lower than the positive ratio of TAP in our physical examination populations. Although the several precancerous patients whose positive TAP might had increased the overall TAP-positive rate, it still indicated that the sensitivity of TAP detection was high, but the specificity was poor.

Our result showed that the positive rate of TAP test in females was higher than that in males, indicating that TAP levels may be affected by the gender. To this end, this study further compared the TAP levels of different genders of physical examination subjects, and found that the levels of TAP in female were significantly higher than those in males, which further indicates that the TAP levels among different gender population with heterogeneous. Therefore, different distinguished standard should be formulated and based on gender to strengthen accuracy.

Further analysis found that the TAP levels in the subjects over 60 were significantly higher than those in younger subjects. Additionally, the TAP level increased with age. Clinically, age was an independent risk factor for many malignancies, the median age of onset in many malignancies is greater than 60 years old ${ }^{[21]}$. The incidence of malignancies has correspondingly increased with age ${ }^{[22,23]}$. The trend changing 
with age in TAP detection was consistent with tumors incidence, which further indicated that TAP detection had higher sensitivity, and also reflected the high reliability of the data in this study.

Pulmonary nodules were common abnormal results in chest XCT examinations. A number of circulating biomarkers and tumor markers, such as plasma microRNAs and circulating cytokines, have been suggested as possible candidates to aid malignant risk assessment for pulmonary nodules, but validation studies are currently lacking ${ }^{[24,25]}$. Clinically, regular follow-up examination is recommended for people with small pulmonary nodules. Chest XCT scan is usually performed in physical examination. The positive rate of pulmonary nodules showed an upward trend in the TAP-positive population, but there was no statistical significance between TAP-positive with TAP-negative populations, and no statistical difference in the number and the sizes of pulmonary nodules. The cause of pulmonary nodules was diverse. Pulmonary nodules may not lead to obvious clinical symptoms in the early stage, but they may become malignant later ${ }^{[26]}$. There was no significant statistical correlation between lung nodules and TAP levels in this study, which may be due to small subject numbers. However, the positive rate of pulmonary nodules in TAP-positive patients was on the rise, suggesting that TAP detection may be one of the indicators for regular follow-up of pulmonary nodule-positive patients.

Clinically, tumor biomarkers ${ }^{[27,28]}$ were commonly used as indicators for detecting malignancies with high sensitivity and specificity. These tumor biomarkers usually tend to be malignancy type specific. Therefore, it is important to find new detection methods that are sensitive to multiple malignancies. Correlation analysis between TAP levels and tumor biomarker levels in this study did not show statistically differences, which indicated that TAP was not a simple extension of the traditional tumor biomarkers, but an independent indicator of tumor screening. TAP detection combined with some variety tumor biomarkers could substantially detect a variety of tumors. TAP detection could screen a variety of malignancies. However, the specificity was poor because TAP level could not reflect one or more malignancies like traditional tumor biomarkers. Thus, it was more suitable for initial screening than for diagnosis.

We had further analyzed the correlation between TAP level and laboratory parameters in physical examination subjects. The study found that there was statistically difference in hemoglobin levels between the TAP positive and the TAP negative subjects. As the diagnostic criteria of hemoglobin were different in gender groups, we also found TAP levels were different between male and female. Further analysis and study on larger samples and mechanisms will be required to determine relationship among gender, hemoglobin and TAP to ascertain the diagnostic criteria.

There were still several limitations in this study. Firstly, different stages of malignant tumor patients might lead to statistical heterogeneous. Secondly, this study showed that there were differences of TAP levels in different gender groups. Due to the large heterogeneity of the patients and the limited of sample size, the accurate of diagnostic criterion in different gender people were insufficient. Thirdly, the number of cases varies greatly among different patients, especially the number of patients with precancerous lesions is small, and the statistical results are less reliable. Finally, this study indicated that there were 
possible links among TAP, gender and hemoglobin. Therefore, we will perform statistical analysis on larger sample data, complete relevant fundamental experiments, and explore related mechanisms in the future.

In conclusion, TAP level was higher in female and elevated with age. TAP was a potential biomarker for screening and following-up of malignancies, which was sensitive to multiple malignancies. There may be some potential links between TAP and hemoglobin, which deserve further investigation.

\section{Declarations}

Ethics approval and consent to participatelThe study has been approved with exempt ethics as a retrospective study by the Ethics Committee of the Second Affiliated Hospital of Soochow University. Due to the retrospective nature of the study, informed consent was waived.

Consent for publication: The study does not involve patient private information.

Availability of data and materials: The datasets used and/or analysed during the current study are available from the corresponding author on reasonable request.

Competing of Interest: The authors declare no conflicts of interest.

Funding: This work was supported by Youth Research Fund of the Second Affiliated Hospital of Soochow University (SDFEYQN1810).

Author Contributions: Conceptualization, Shuhua Wu and Lin Zhang; methodology, Changjun Tian and Zhihui Zhang; formal analysis, Hui Fan and Yongsheng Zhang; original draft preparation, Zhihui Zhang and Bai Li; writing-Review and editing, Mingxin Gao and Linfang Li; visualization, Linfang Li; supervision, Shuhua Wu; funding acquisition, Shuhua Wu. All authors have read and agreed to the published version of the manuscript.

Acknowledgments: Thanks for the support and help of the Department of Geriatrics, Physical Examination Center and Pathology Department of the Second Affiliated Hospital of Soochow University.

\section{References}

1. Mereiter S, Balmaña M, Campos D, et al. Glycosylation in the Era of Cancer-Targeted Therapy: Where Are We Heading? Cancer Cell. 2019;36(1):6-16.

2. Sun C, Deng F, Meng L, et al. Correlation between TAP detection and common digestive tract precancerous lesions. Oncol Lett. 2018;15(2):1616-20.

3. Liu Z, Cai J, Yu Y, et al. Tumor Abnormal Protein as a Novel Biomarker in Papillary Thyroid Carcinoma. Clin Lab. 2017;63(3):479-85. 
4. Ma A, Fan D, Yan F. A study of the application of TAP combined with transvaginal ultrasound in the diagnosis of early-stage endometrial cancer. Oncol Lett. 2018;16(4):5186-90.

5. Zhang L, Guo X, Min Y, et al. Tumor abnormal protein (TAP) examination contributes to primary diagnosis of bladder cancer. Int J Clin Exp Med. 2015;8(10):18528-32.

6. Li LX, Zhang B, Gong RZ. Insights into the role of tumor abnormal protein in early diagnosis of cancer: A prospective cohort study. Med (Baltim). 2020;99(11):e19382.

7. Graham TA, Sottoriva A. Measuring cancer evolution from the genome. J Pathol. 2017;241(2):18391.

8. Ferlay J, Colombet M, Soerjomataram I, et al. Cancer incidence and mortality patterns in Europe: Estimates for 40 countries and 25 major cancers in 2018. Eur J Cancer. 2018;103:356-87.

9. Lanier JB, Park JJ, Callahan RC. Anemia in Older Adults. Am Fam Physician. 2018;98(7):437-42.

10. Murphy WG. The sex difference in haemoglobin levels in adults - mechanisms, causes, and consequences. Blood Rev. 2014;28(2):41-7.

11. Bosman FT, Carneiro F, Hruban RH, et al (2010) WHO classification of tumours of the digestive system. Lyon (France): International Agency for Research on Cancer. (WHO classification of tumours series, 4th ed.; vol. 3).

12. Lloyd RV, Osamura RY, Klöppel G, et al (2017) WHO classification of tumours of endocrine organs. Lyon (France): International Agency for Research on Cancer. (WHO classification of tumours series, 4th ed.; vol. 10).

13. Moch $\mathrm{H}$, Humphrey PA, Ulbright TM, et al. WHO Classification of Tumours of the Urinary System and Male Genital Organs. Lyon: International Agency for Research on Cancer; 2016.

14. Louis DN, Perry A, Reifenberger G, et al. The 2016 World Health Organization classification of tumors of the central nervous system: a summary. Acta Neuropathol. 2016;131:803-20.

15. Travis WD, Brambilla E, Burke AP, et al. WHO Classification of Tumours of the Lung, Pleura, Thymus and Heart. 4th ed. Lyon: IARC; 2015.

16. Hong QY, Wu GM, Qian GS, et al. Prevention and management of lung cancer in China. Cancer. 2015;121(17):3080-8.

17. Rugge $M$, Meggio A, Pravadelli $C$, et al. Gastritis staging in the endoscopic follow-up for the secondary prevention of gastric cancer: a 5-year prospective study of 1755 patients. Gut. 2019;68(1):11-7.

18. Byers $T$, Wender RC, Jemal A, et al. The American Cancer Society challenge goal to reduce US cancer mortality by 50\% between 1990 and 2015: results and refflections. CA Cancer J Clin. 2016;66:35969.

19. Shieh Y, Eklund M, Sawaya GF, et al. Population-based screening for cancer: hope and hype. Nat Rev Clin Oncol. 2016;13:550-65.

20. Esserman LJ, Thompson IM, Reid B, et al. Addressing overdiagnosis and overtreatment in cancer: a prescription for change. Lancet Oncol. 2014;15:e234-42. 
21. Siegel RL, Miller KD, Jemal A. Cancer statistics, 2019. CA:cancer J Clin. 2019;69:7-34.

22. Chen $T$, Zhou $F$, Jiang W, et al. Age at diagnosis is a heterogeneous factor for non-small cell lung cancer patients. J Thorac Dis. 2019;11(6):2251-66.

23. Miller KD, Nogueira L, Mariotto AB, et al. Cancer Treatment and Survivorship Statistics, 2019. CA Cancer J Clin. 2019;69(5):363-85.

24. Shen J, Liu Z, Todd NW, et al. Diagnosis of lung cancer in individuals with solitary pulmonary nodules by plasma microRNA biomarkers. BMC Cancer. 2011;11:374.

25. Daly S, Rinewalt D, Fhied C, et al. Development and validation of a plasma biomarker panel for discerning clinical signifificance of indeterminate pulmonary nodules. J Thorac Oncol. 2013;8:31-6.

26. Nemesure B, Clouston S, Albano D, et al. Will That Pulmonary Nodule Become Cancerous? A Risk Prediction Model for Incident Lung Cancer. Cancer Prev Res (Phila). 2019;12(7):463-70.

27. Guo B, Lian W, Liu S, et al. Comparison of diagnostic values between CA125 combined with CA199 and ultrasound combined with CT in ovarian cancer. Oncol Lett. 2019;17(6):5523-8.

28. Holdenrieder S, von Pawel J, Dankelmann E, et al. Nucleosomes, ProGRP, NSE, CYFRA 21 - 1, and CEA in monitoring first-line chemotherapy of small cell lung cancer. Clin Cancer Res. 2008;23:781321.

\section{Figures}




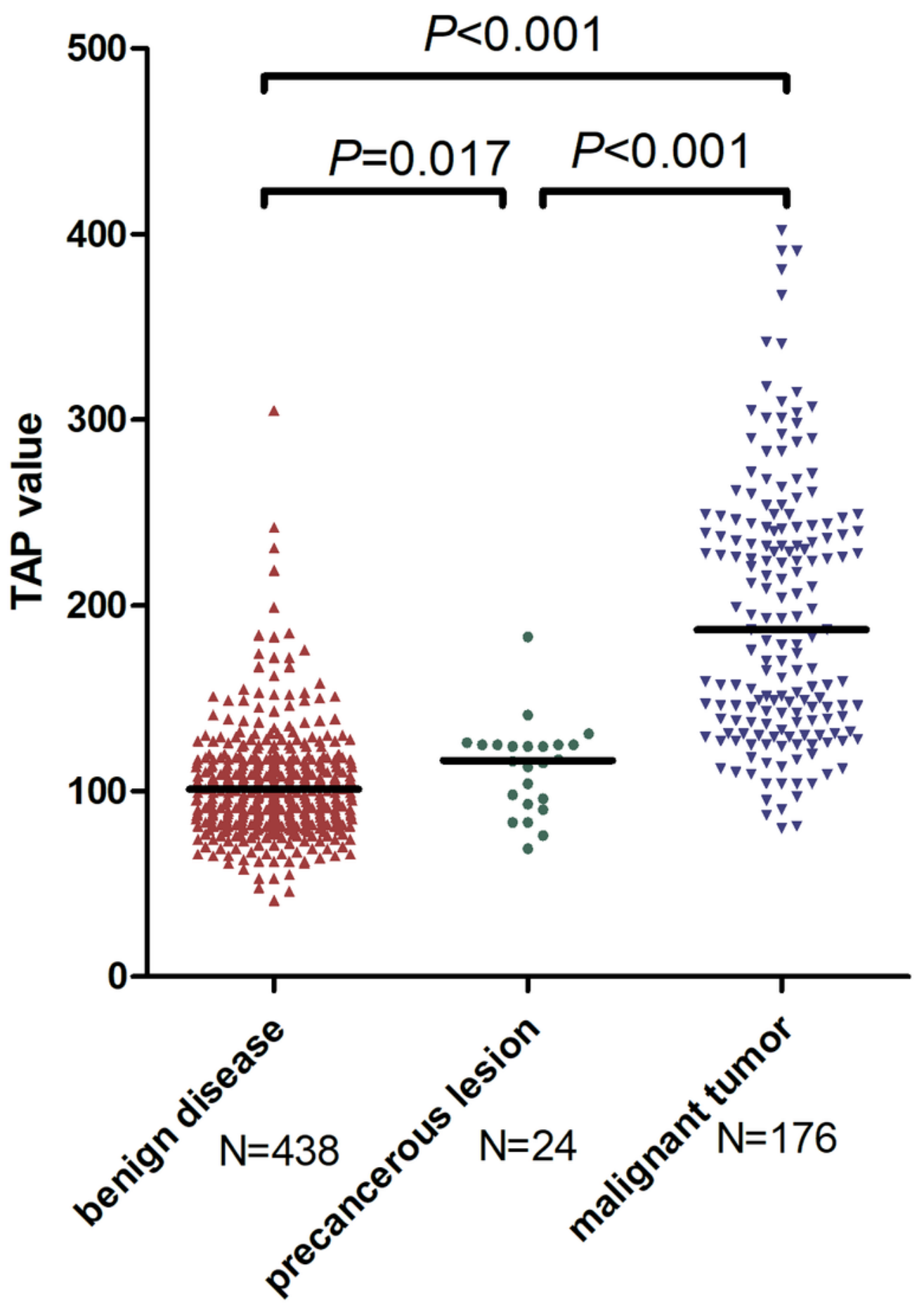

Figure 1

TAP levels in patients with benign disease, precancerous, and malignancies. 


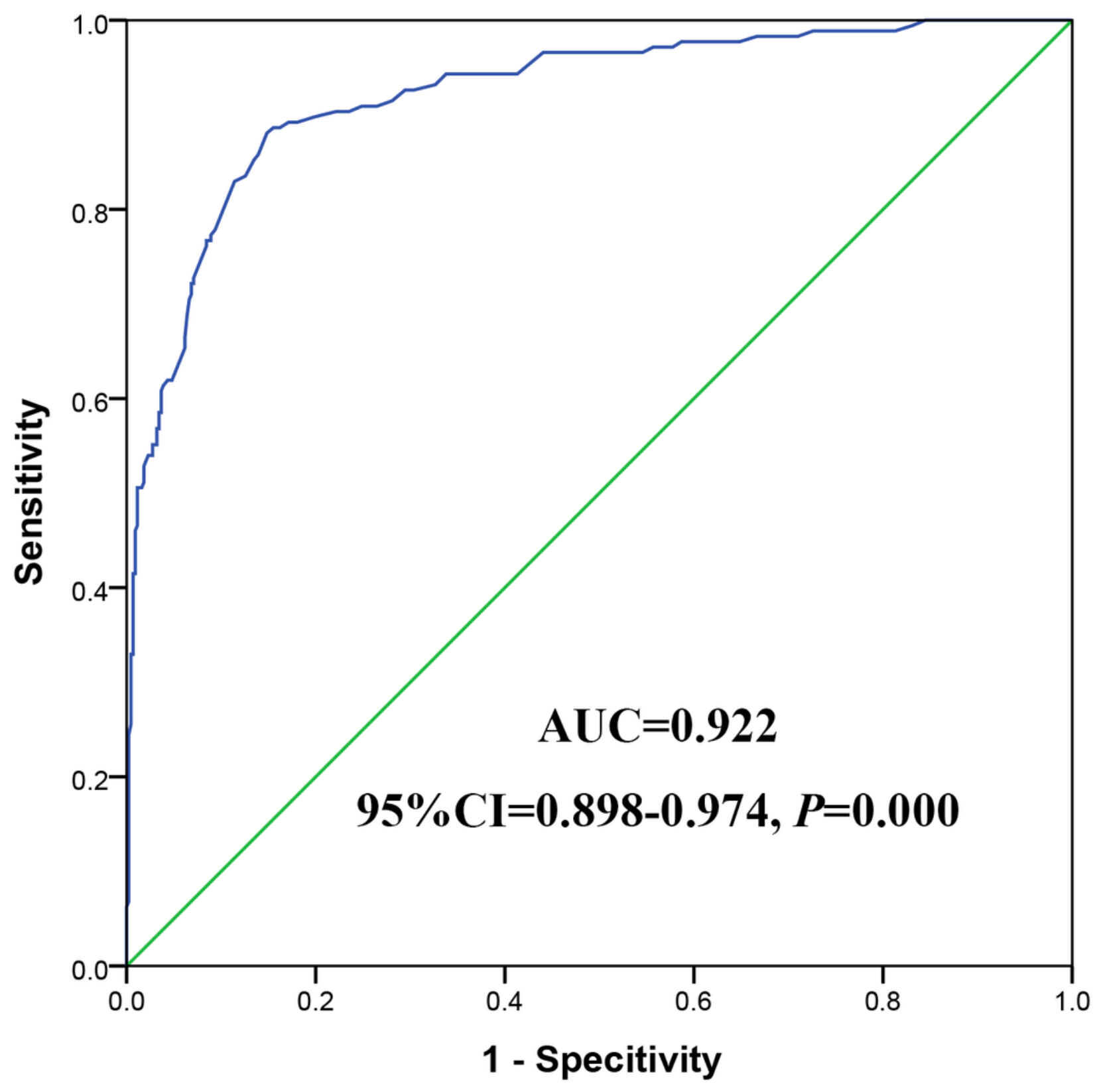

Figure 2

TAP level was an effective indicator to differentiate malignancies from benign diseases (When the Yoden index (sensitivity + specificity -1) was the largest, the cut-off value of TAP level was 124.50, with the ability to distinguish between malignancies and benign diseases.) 


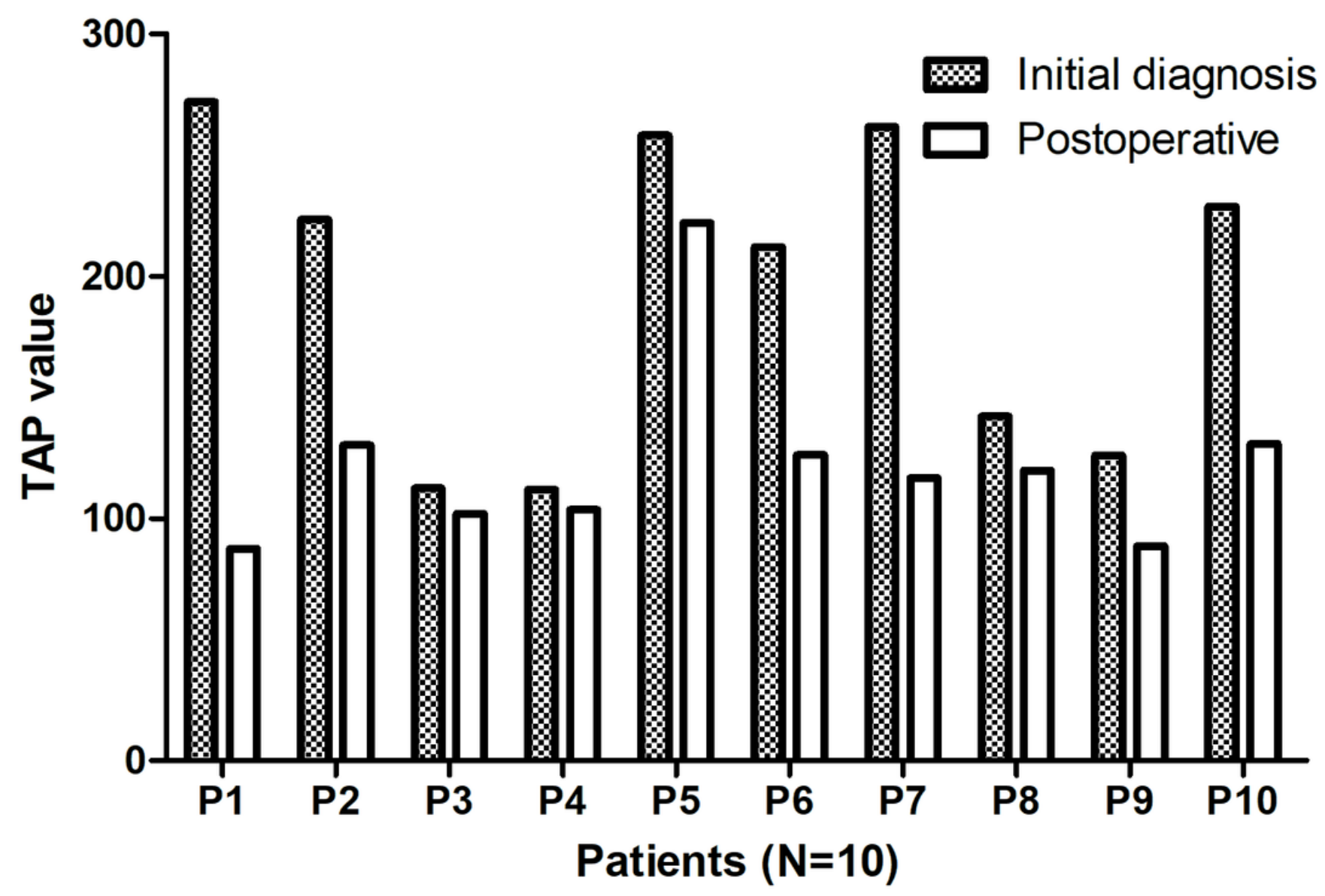

Figure 3

The changing of TAP levels in malignant patients from initial diagnosis to post operation (Randomly selected initial diagnosed patients for dynamic monitoring, there was a significantly decrease of TAP levels in malignant patients after surgery.) 


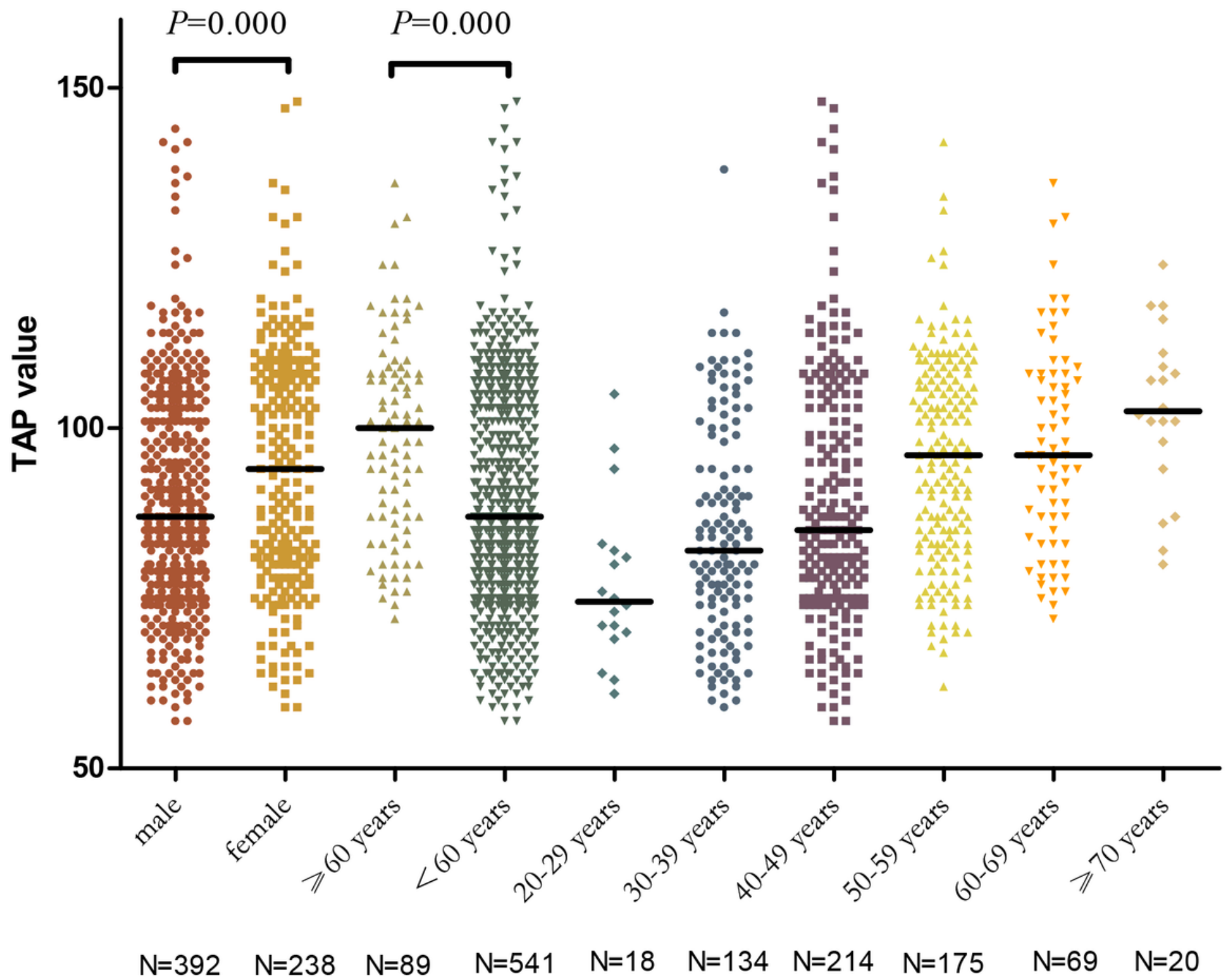

Figure 4

TAP levels in different genders and age gradient groups (TAP levels were higher in female physical examination subjects, and also increased with age.) 